\title{
First and third trimester serum concentrations of adropin and copeptin in gestational diabetes mellitus and normal pregnancy
}

\author{
Filip A. Dąbrowski ${ }^{1}$, Patrycja Jarmużek ${ }^{1}$, Agata Gondek², Agnieszka Cudnoch-Jędrzejewska², \\ Dorota Bomba-Opoń ${ }^{1}$, Mirosław Wielgoś ${ }^{1}$ \\ ${ }^{1} 7^{\text {st }}$ Department of Obstetrics and Gynecology, Medical University of Warsaw, Poland \\ ${ }^{2}$ Department of Experimental and Clinical Physiology, Laboratory of Centre for Preclinical Research, \\ Medical University of Warsaw, Poland
}

\begin{abstract}
Objectives: Gestational diabetes mellitus (GDM) is a metabolic disease diagnosed in $1.7 \%$ up to $11.6 \%$ pregnancies. The prevalence of adverse pregnancy outcome is significantly higher in the case of early onset of diabetes mellitus. Adropin is a hormone promoting carbohydrate oxidation over fat oxidation, and influence nitric oxide synthase. Copeptin is a cleavage product of the vasopressin precursor recently correlated with diabetes mellitus. The aim of the study was to determine maternal serum adropin and copeptin concentrations in women with early and late manifestation of GDM and to discuss their potential role as biochemical markers of insulin resistance.

Material and methods: Case-control study on 58 pregnant Caucasian women. Serum levels of adropin and copeptin were assessed in patients with early onset (GDM1) and classical gestational diabetes mellitus (GDM2). Complications such as macrosomia and hypotrophy were evaluated.

Results: There was no significant difference between the study and the control group (age, BMI, parity). Fetal growth disturbance rate was $37.5 \%$ in GDM1, $11 \%$ in GDM2 and $6 \%$ in controls. Adropin concentration in GDM patients was significantly higher than in control group $(p<0.001)$, but there was no difference between GDM1 and GDM2 group. High serum concentration of adropin positively correlated with elevated $\mathrm{HbA}_{1 c}(p<0.05)$. The groups did not differ in terms of copeptin serum concentration.

Conclusions: High adropin serum concentration in GDM patients is associated with increased risk of fetal growth disturbances, possibly due to improper placentation. According to our prospective study, neither copeptin nor adropin serum concentration are useful to discriminate between early and late onset of gestational diabetes mellitus.
\end{abstract}

Key words: pregnancy, diabetes mellitus, gestational diabetes mellitus, adropin, copeptin

Ginekologia Polska 2016; 87, 9: 629-634

\section{INTRODUCTION}

Gestational diabetes mellitus (GDM) is a metabolic disease defined as hyperglycemia with blood glucose values above normal, but below those diagnostic for diabetes, with its onset or being first diagnosed during pregnancy. The prevalence of GDM varies between populations, ranging from $1.7 \%$ to $11.6 \%$ [1]. Numerous studies found that GDM is associated with significantly higher maternal (cesarean section, gestational hypertension, preeclampsia), perinatal (macrosomia and birth injury) and postnatal (early onset of metabolic syndrome, diabetes and hypertension in children) complications risk [2,3]. The prevalence of adverse pregnancy outcome is significantly higher in the case of early onset of diabetes mellitus.

Throughout pregnancy, maternal glucose metabolism undergoes multiple, adaptive changes to ensure adequate fetal development. In conditions of increased glucose utilization by feto-placental unit and high level of human placental lactogen, placenta-derived human growth factor hormone, progesterone, cortisol, prolactin and many other 
hormones, decreasing insulin sensitivity occurs in peripheral tissues [4]. As a compensation, both maternal hepatic gluconeogenesis and fatty acid levels increase [5]. Accompanied by certain environmental and genetic factors, maternal metabolism is not able to properly compensate increasing insulin resistance and together with the impaired insulin secretion and higher level of hepatic gluconeogenesis result in elevated glycaemia [6].

Adropin (ADR) is a 42-amino-acid peptide hormone that participates in maintaining glucose homeostasis. Recently, a novel role for ADR was reported in promoting carbohydrate oxidation over fat oxidation in skeletal muscles [7]. Moreover, adropine's influence on expression of an inducible nitric oxide synthase was discovered [8], which can explain ADRs' potential role in prediction of endothelial dysfunction in patients with diabetes mellitus [9].

Several reports suggest that stress-mediated activation of the HPA axis, accompanied by increased plasma levels of arginine vasopressin (AVP) may play a crucial role in pathogenesis of insulin resistance and metabolic syndrome [10]. AVP binds with three different receptors: V1aR, V1bR and V2R. Activation of V1aR in hepatocytes increases glycogenolysis, whereas binding with $\mathrm{V} 1 \mathrm{bR}$ in pituitary gland and pancreas lead to insulin and glucagon secretion [11, 12]. Copeptin is a cleavage product of the $\mathrm{C}$-terminal part of the AVP precursor, stable and with long half-life, which is also known as a surrogate hormone for AVP. In recent studies, the correlation with elevated plasma copeptin and diabetes mellitus has been investigated [13].

Although there are many similarities between early and late onset of diabetes, in both cases the underlying pathophysiology remains unclear. For many decades, a lot of effort has been made to find biochemical markers, which would improve screening for GDM, with no satisfactory result.

\section{OBJECTIVES}

To the best of our knowledge, this is one of very few studies that investigate adropin and copeptin serum concentration in women with early and late onset of GDM. The aim of this study was to investigate whether maternal serum adropin and copeptin concentrations are altered in women with early and late manifestation of GDM and to discuss their potential role as biochemical markers of insulin resistance.

\section{MATERIAL AND METHODS}

This case-control study was performed from July 2014 until August 2015 in $1^{\text {st }}$ Department of Obstetrics and Gynecology, Medical University of Warsaw. An informed consent was obtained from all patients on forms approved by the Ethics Committee of Medical University of Warsaw. Fifty eight Caucasian women were recruited, 20 with GDM diagnosed in the $1^{\text {st }}$ trimester (GDM1 group), 20 with clas- sical GDM (GDM2 group) and 18 controls (noGDM1 and noGDM2 group) with uncomplicated pregnancies. Blood samples were collected in the $1^{\text {st }}$ (GDM1 and noGDM1 group) and $3^{\text {rd }}$ trimester (GDM2 and noGDM2) for examinations in Department of Experimental and Clinical Physiology, Medical University of Warsaw. All patients gave written informed consent before the study began.

GDM was defined as fasting glucose $>5.11 \mathrm{mmol} / \mathrm{L}$ and/or OGTT results $0 \mathrm{~h}>5.11,1 \mathrm{~h}>10,2 \mathrm{~h}>8.5 \mathrm{mmol} / \mathrm{L}$. All GDM patients received education in carbohydrates restriction diet, exercise and glycaemia monitoring. They kept a journal of food intake and glycaemia levels (4 to 9 point). Thanks to careful diet, blood glucose levels were within normal range (fasting $<5.28 \mathrm{mmol} / \mathrm{L}$, postprandi$\mathrm{al}<7.22 \mathrm{mmol} / \mathrm{L}$ ) and none of them needed insulin treatment. Except for GDM status, pregnancy complications such as cesarean section, macrosomia, and hypotrophy were evaluated.

Exclusion criteria were as follows: preeclampsia, a history of fetal anomalies, chronic diseases (e.g. AIDS, hepatitis, renal insufficiency, and heart disease), premature rupture of membranes prior to 24th week, intrauterinal infection. Gestational age was estimated by the last menstrual period and was confirmed by $1^{\text {st }}$ trimester ultrasound. Women from the control group had no significant medical history, no pregnancy-related complications and no risk factors for gestational diabetes. $1^{\text {st }}$ trimester fasting glucose and a $75 \mathrm{~g}$ 3-point glucose tolerance test in $3^{\text {rd }}$ trimester values were within normal range.

At the time of GDM diagnosis, serum samples were collected from pregnant women. All samples were kept at room temperature for at least $60 \mathrm{~min}$ to allow the blood clot formation and were later centrifuged at 20000 RPM for $5 \mathrm{~min}$ and kept frozen in $-80^{\circ}$ Celsius. Serum copeptin concentrations were measured by enzyme immunoassay (EIA) using the following commercially available reagent kits: Copeptin (Human) — ElA Kit; kit Extraction free, Phoenix Pharmaceuticals, INC; Minimum Detectable Concentration $=0.12 \mathrm{ng} / \mathrm{mL}$. Serum adropin concentrations were measured by enzyme-linked immunosorbent assay (ELISA) using the following commercially available reagent kits: Human (AD) ELISA Kit, Sunred Biological Technology Co., Shanghai, Minimum Detectable Concentration $=0.306 \mathrm{ng} / \mathrm{mL}$.

All statistical analyses were performed using the GraphPad, MS Office Excel 2010 and Statistica 10 software. Data were presented as mean \pm standard deviation. Mean values between the groups were compared by Student's T-test. Pearson's correlation coefficient was used to determine the relationship between the variables. Power of the statistical test, which the compared groups were analysed with, ranged from 0.739 to 0.996 . In the calculation of power of the test as a criterion of the quantity, minimum $n$ in the groups 
has been assumed. All reported $p$ values are two-tailed and a $p<0.05$ was considered significant.

\section{RESULTS}

There was no statistical difference between the study and the control patients in terms of age, blood pressure, gestational age at delivery and weight gain (Tab. 1). Patients who developed GDM were significantly more overweight before pregnancy. In GDM1, body mass index (BMI) was $26.33 \pm 6.42$, and in GDM2 $28.26 \pm 5.99$, while in controls $-22.9 \pm 4.1 \mathrm{~kg} / \mathrm{m}^{2}(\mathrm{p}<0.001)$.

Comparison of BMI between GDM1, GDM2 and control groups are described in Figure 1 and comparison of serum concentration of fasting glucose, adropin and copeptin between GDM1 and GDM2 groups are described in Figure 2. Figure 3 presents differences in distribution of those characteristics between controls and diabetic patients. Adropin concentration in GDM patients $(11.78 \pm 1.09 \mathrm{ng} / \mathrm{ml})$ was significantly higher than in control group $(5.68 \pm 1.27 \mathrm{ng} / \mathrm{mL}, \mathrm{p}<0.001)$, but there was no difference between GDM1 $(12.71 \pm 1.79 \mathrm{ng} / \mathrm{mL})$ and GDM2 (11.12 $\pm 1.39 \mathrm{ng} / \mathrm{mL})$ group.

We did not find statistically important differences between GDM patients versus controls in neonatal weight at delivery (42.9 pc in GDM1 $\pm 33.9,52.1$ pc \pm 26.1 in GDM2, and $41.2 \mathrm{pc} \pm 24.7$ in controls). Interestingly, the cesarean section rate was higher in control group $(p<0.05)$.

Although the difference in neonatal weight was not statistically significant, it is worth to note that there were 6 cases $(37.5 \%)$ in GDM1 group of growth disturbances (3 hypotrophic $10 \mathrm{pc}$, and 3 macrosomic > $90 \mathrm{pc}$ ) vs. only 2 macrosomic neonates in GDM2 (11\%) and only 1 hypotrophic child in no GDM patients (6\%).

Adropin serum concentration in diabetic patients was significantly higher than in control group ( $p>0.001)$, but there was no difference in copeptin serum concentration (Tab. 2).

Analysis of correlation found no link between adropin and copeptin serum concentration and fasting glucose or $B M I$. We found a significant correlation between high serum concentration of adropin and elevated $\mathrm{HbA}_{1 c}(p<0.05)$, suggesting long lasting disease and possible carbohydrate disturbances prior to pregnancy.

\section{DISCUSSION}

The key finding of our study is that maternal serum adropin in women with GDM, both diagnosed in the first and third trimester, is significantly elevated in respect to non-diabetic

\section{Table 1. Characteristics of the patients}

\begin{tabular}{|l|c|c|c|}
\hline Characteristic & GDM1 & GDM2 & Control \\
\hline BMI before pregnancy $\left[\mathrm{kg} / \mathrm{m}^{2}\right]$ & $28.20 \pm 7.78$ & $24.45 \pm 4.10$ & $22.90 \pm 4.10$ \\
\hline BMl before delivery $\left[\mathrm{kg} / \mathrm{m}^{2}\right]$ & $28.41 \pm 7.55$ & $28.09 \pm 4.07$ & $27.20 \pm 4.66$ \\
\hline Fasting glucose $1^{\text {st }}$ trimester $[\mathrm{mmol} / \mathrm{L}]$ & $5.50 \pm 0.65$ & $4.95 \pm 0.31$ & $4.37 \pm 0.37$ \\
\hline \% tile at delivery $[\mathrm{no}]$. & $42.88 \pm 33.93$ & $52.06 \pm 26.10$ & $41.20 \pm 24.68$ \\
\hline
\end{tabular}

$\mathrm{BMI}$ - body mass index; GDM1 - gestational diabetes mellitus diagnosed in the $1^{\text {st }}$ trimester; GDM2 - gestational diabetes mellitus diagnosed in the $3^{\text {rd }}$ trimester

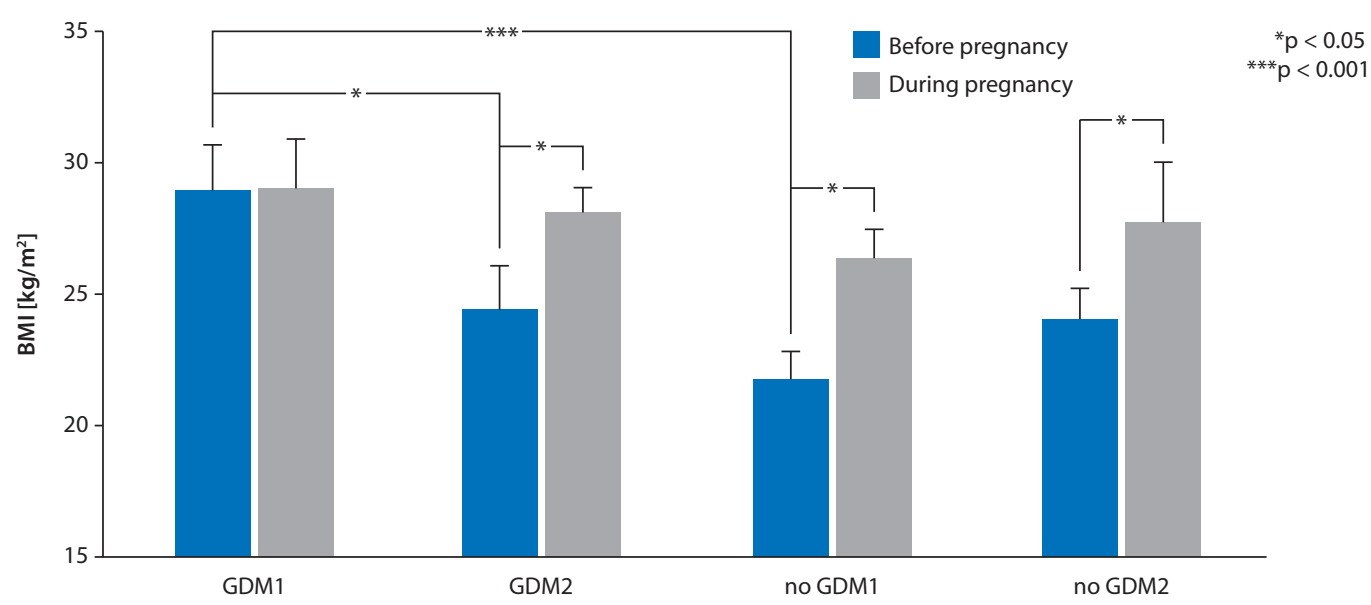

Figure 1. Body mass index (BMI) in patients with gestational diabetes mellitus diagnosed in the $1^{\text {st }}(\mathrm{GDM} 1)$ and $3^{\text {rd }}(\mathrm{GDM} 2)$ trimester and control groups (no GDM1, no GDM2) before and during pregnancy 

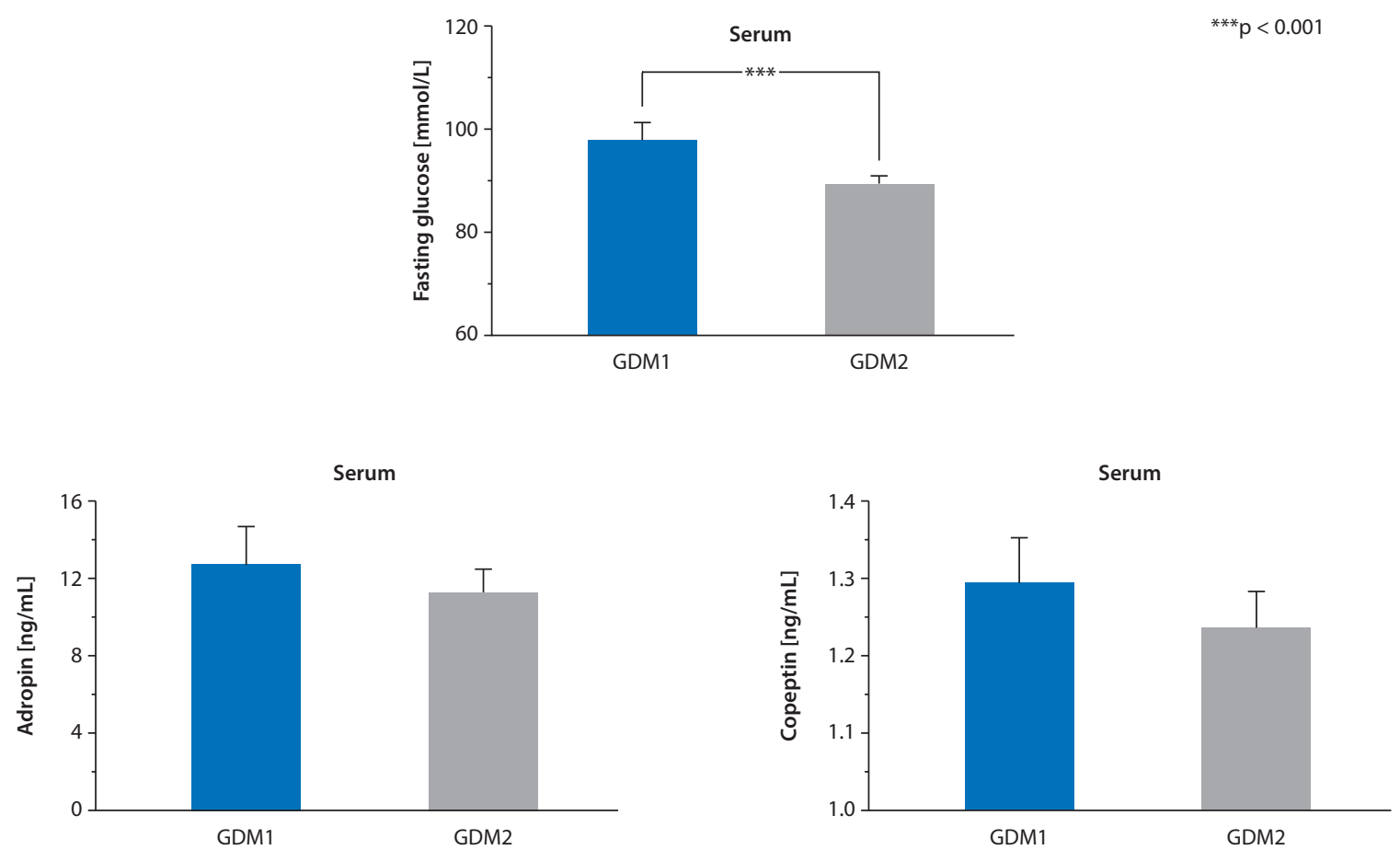

Figure 2. Serum concentration of fasting glucose, adropin and copeptin in patients with gestational diabetes mellitus diagnosed in the $1^{\text {st }}$ (GDM1) and $3^{\text {rd }}(\mathrm{GDM} 2)$ trimester
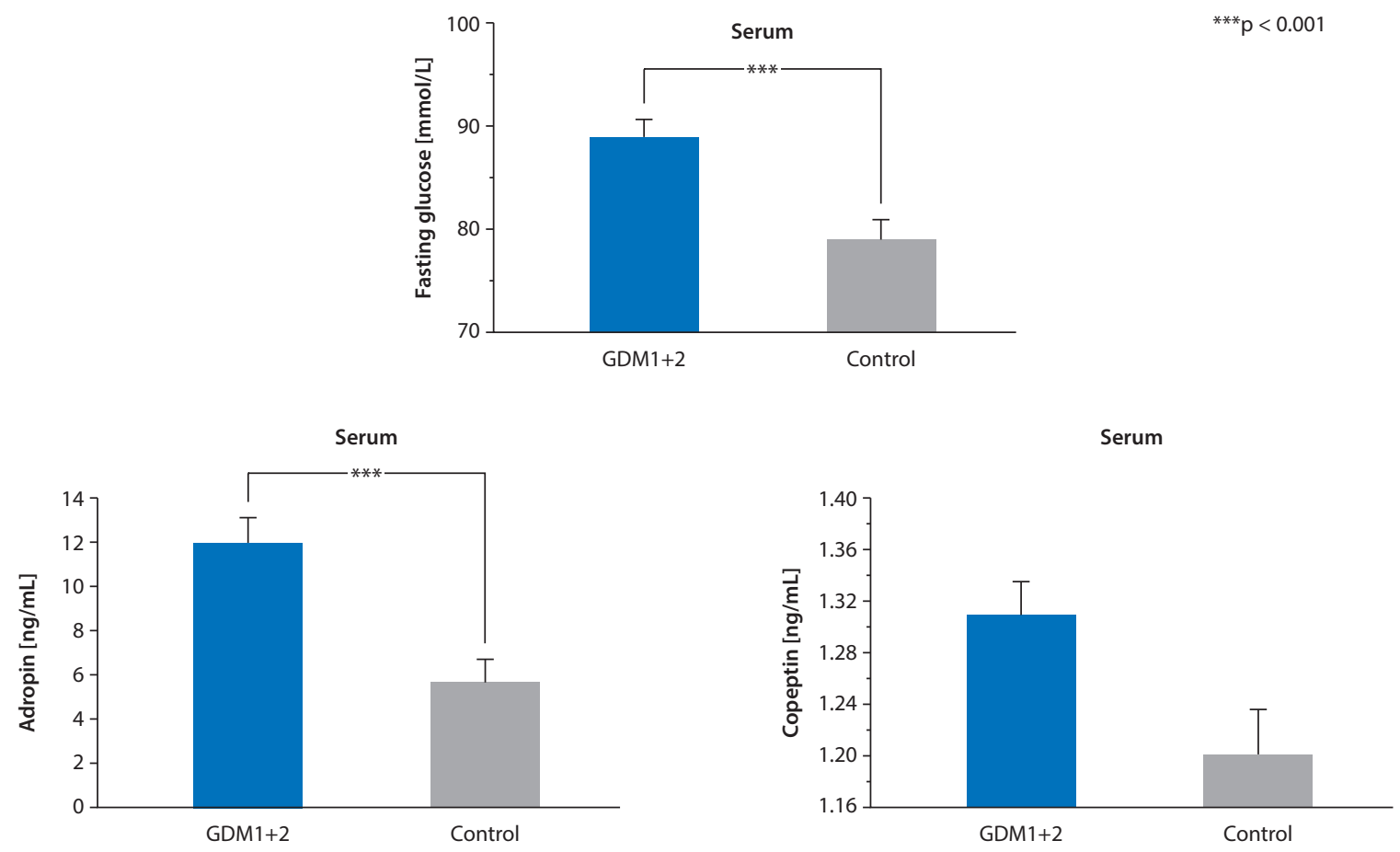

Figure 3. Serum concentration of fasting glucose, adropin and copeptin in diabetic patients (pooled group GDM1+2) and control group

pregnant women. Adropin is encoded by the Energy Homeostasis Associated (Enho) gene that is widely expressed in the central nervous system, as well as peripheral tissues such as liver, the heart and skeletal muscles [14-16]. While the mechanism of release remains controversial, the rapid regulation according to energy and nutritional status has been observed. Gao Su et al. (2015) in their study on obese mice with insulin resistance suggest the therapeutic effects of adropin on glucose tolerance. They concluded that adropin improves glucose clearance, ameliorates insulin resistance 
Table 2. Comparison of adropin, copeptin and $\mathrm{HbA}_{1 \mathrm{c}}$ serum concentrations between groups

\begin{tabular}{|l|c|c|c|c|}
\hline Serum & GDM1 & GDM2 & GDM1+2 & Control \\
\hline Adropin $[\mathrm{ng} / \mathrm{ml}]$ & $12.71 \pm 1.79$ & $11.12 \pm 1.39$ & $11.78 \pm 1.09^{*}$ & $5.68 \pm 1.27^{*}$ \\
\hline Copeptin $[\mathrm{ng} / \mathrm{ml}]$ & $1.32 \pm 0.13$ & $1.31 \pm 0.15$ & $1.31 \pm 0.12$ & $1.20 \pm 0.14$ \\
\hline $\mathrm{HbA}_{1 \mathrm{c}}[\mathrm{mmol} / \mathrm{L}]$ & $5.12 \pm 0.28$ & $5.20 \pm 0.55$ & $5.16 \pm 0.41$ & NA \\
\hline
\end{tabular}

$\mathrm{HbA}_{1 \mathrm{c}}$ - glycated hemoglobin; GDM1 - gestational diabetes mellitus diagnosed in the $1^{\text {st }}$ trimester; GDM2 - gestational diabetes mellitus diagnosed in the $3^{\text {rd }}$ trimester; GDM1+2 - pooled GDM1 and GDM2 group; NA — not available; ${ }^{*} \mathrm{p}<0.001$

and reverses dyslipidemia. Moreover, increased release of adropin is related to the fed state and increased glucose oxidation by pyruvate dehydrogenase (PDH) complex activation. Furthermore, adropin inhibits carnitine palmitoyltransferase-1B (CPT1B), which reduces fatty acid oxidation (FAO) in muscles [17]. According to recent studies, it can be speculated that higher concentration of adropin in women with GDM is one of the multiple adaptive responses on adverse glucose metabolism during pregnancy. The use of adropin as a biochemical marker for insulin resistance can be considered. In the study of Topuz et al., adropin and hemoglobin $A_{1 c}$ were deemed independent risk factors for endothelial dysfunction in individuals with type 2 diabetes mellitus [9]. In pregnancy, endothelial dysfunction can contribute to the impaired placentation in the $1^{\text {st }}$ trimester and higher prevalence of late gestational complications such as preeclampsia, fetal growth disturbance, placental abruption or intrauterine fetal death. In our study group, the rate of pregnancy complications related with placentation disturbance did not reach statistically significant superiority. To investigate the influence of maternal adropin concentration on placentation in early pregnancy further studies are required.

In the recent literature, also mainly discussed is an increased concentration of serum copeptin in pregnant women in relation to preeclampsia or fetal growth restrictions [18]. According to one of the few studies on pregnant women with GDM, a significant correlation between copeptin and HOMA-IR was found [19]. An association between elevated copeptin and increased risk of type 2 diabetes mellitus later in file was observed [20]. In the population-based study conducted in Sweden, copeptin was an independent predicting factor for diabetes mellitus [21]. In an animal study, elevated AVP level as a result of AVP resistance at the level of V1aR was related to insulin resistance and diabetes mellitus $[22,23]$. However, a recent study of Yeung and coauthors (2014) investigates serum copeptin levels during pregnancy in relation to multiple pregnancy complications risk. There, in one of the largest reports, association between increased copeptin concentration and occurrence of preeclampsia were confirmed and no correlation to gestational diabetes was found [24]. Likewise in our study, an elevated serum concentration of copeptin in GDM patients, both in the first and in the third trimester, was not statistically significant. In conclusion, although copeptin seems to play an important role in AVP system and can be useful in screening for preeclampsia, its role as a marker for insulin resistance and GDM remains questionable. However, it should be noted that our study had limitation. The sample size for GDM patients was relatively small.

Adropin and copeptin affect glucose homeostasis. Available literature reports few discrepant results concerning adropin and copeptin blood concentration in women with gestational diabetes mellitus. Although the levels in those pregnant patients are affected by GDM change, their influence on insulin resistance in pregnancy needs further investigation.

\section{CONCLUSIONS}

High adropin serum concentration in GDM patients is associated with increased risk of fetal growth disturbances, possibly due to improper placentation. According to our prospective study, neither copeptin nor adropin serum concentration are useful to discriminate between early and late onset of gestational diabetes mellitus.

\section{REFERENCES}

1. Schneider S, Block C, Wetzel M, [et al.]. The prevalence of gestational diabetes in advanced economies. J Perinat Med. 2012, 40, 511-520.

2. Nicholas LM, Morrison JL, Rattanatray L, [et al.]. The early origins of obesity and insulin resistance: timing, programming and mechanisms. Int J Obes. (Lond) 2016, 40, 229-238.

3. Srichumchit S, Luewan S, Tongsong T. Outcomes of pregnancy with gestational diabetes mellitus. Int J Gynaecol Obstet. 2015, 131, 251-254.

4. Newbern D, Freemark M. Placental hormones and the control of maternal metabolism and fetal growth. Curr Opin Endocrinol Diabetes Obes. 2011, 18, 409-416.

5. Di Cianni G, Miccoli R, Volpe L, [et al.]. Intermediate metabolism in normal pregnancy and in gestational diabetes. Diabetes Metab Res Rev. 2003, 19, 259-270.

6. Butte NF. Carbohydrate and lipid metabolism in pregnancy: normal compared with gestational diabetes mellitus. Am J Clin Nutr. 2000, 71 (suppl), 1256S-1261S.

7. Gao S, McMillan RP, Jacas J, [et al.]. Regulation of substrate oxidation preferences in muscle by the peptide hormone adropin. Diabetes. 2014, 63, 3242-3252.

8. Kuloglu T, Aydin S. Immunohistochemical expressions of adropin and Inducible nitric oxide synthase in renal tissues of rats with streptozotocin-ınduced experimental diabetes. Biotech Histochem. 2014, 89, 104-110.

9. Topuz M, Celik A, Aslantas T, [et al.]. Plasma adropin levels predict endothelial dysfunction like flow-mediated dilatation in patients with type 2 diabetes mellitus. J Investig Med. 2013, 61, 1161-1164.

10. Bjorntorp P, Rosmond R. Hypothalamic origin of the metabolic syndrome X. Ann NY Acad Sci. 1999, 892, 297-307. 
11. Keppens $\mathrm{S}$, de Wulf $\mathrm{H}$. The nature of the hepatic receptors involved in vasopressin-induced glycogenolysis. Biochim Biophys Acta. 1979, $588,63-69$.

12. Abu-Basha EA, Yibchok-Anun S, Hsu WH. Glucose dependency of arginine vasopressin-induced insulin and glucagon release from the perfused rat pancreas. Metabolism. 2002, 51, 1184-1190.

13. Saleem U, Khaleghi M, Morgenthaler NG, [et al.]. Plasma carboxy-terminal provasopressin (copeptin): a novel marker of insulin resistance and metabolic syndrome. J Clin Endocrinol Metab. 2009, 94, 2558-2564.

14. Kumar KG, Trevaskis JL, Lam DD, [et al.]. Identification of adropin as a secreted factor linking dietary macronutrient intake with energy homeostasis and lipid metabolism. Cell Metabolism. 2008, $8,468-481$.

15. Wong C-M, Wang Y, Lee JTH., Huang, [et al.]. Adropin is a brain membrane-bound protein regulating physical activity via the NB-3/Notch signaling pathway in mice. Journal of Biological Chemistry. 2014, 289, 25976-25986.

16. Lovren F, Pan Y, Quan A, [et al.]. Adropin is a novel regulator of endothelial function. Circulation. 2010, 122, S185-S192.
17. Gao S, McMillan RP, Zhu Q, [et al.]. Therapeutic effects of adropin on glucose tolerance and substrate utilization in diet-induced obese mice with insulin resistance. Mol Metab. 2015, 17, 4, 310-324.

18. Bülbül GA, Kumru S, Erol O, [et al.]. Maternal and umbilical cord copeptin levels in pregnancies complicated by fetal growth restriction. J Matern Fetal Neonatal Med. 2014, 18, 1-7.

19. Oncul M, Tuten A, Kucur M, [et al.]. Copeptin concentrations are not elevated in gestational diabetes mellitus. Arch Gynecol Obstet. 2013, 288, 1045-1049.

20. Enhörning S, Wang TJ, Nilsson PM, [et al.]. Plasma copeptin and the risk of diabetes mellitus. Circulation. 2010, 18, 121, 2102-2108.

21. Canivell S, Ponte B, Pruijm M, [et al.].Plasma copeptin is associated with insulin resistance in a swiss population-based study. JHypertens. 2015, 33 (suppl 1).

22. Aoyagi T, Birumachi J, Hiroyama M, [et al.]. Alteration of glucose homeostasis in V1a vasopressin receptor-deficient mice. Endocrinology. 2007, 148, 2075-2084.

23. Fujiwara $Y$, Hiroyama M, Sanbe A, [et al.]. Insulin hypersensitivity in mice lacking the V1b vasopressin receptor. J Physiol. 2007, 584, 235-244.

24. Yeung EH, Liu A, Mills JL, [et al.]. Increased levels of copeptin before clinical diagnosis of preelcampsia. Hypertension. 2014, 64, 1362-1367. 